\title{
Analysis of fractional-order Chen System and Its application in Image Encryption
}

\author{
Hongyan Jia \\ Department of Automation, Tianjin University of Science and Technology, \\ 1038 Dagunanlu Road, Hexi District, Tianjin300222, PR China \\ Qinghe Wang \\ Department of Automation, Tianjin University of Science and Technology, \\ 1038 Dagunanlu Road, Hexi District, Tianjin 300222, PR China \\ E-mail: jiahy@tust.edu.cn \\ www.tust.edu
}

\begin{abstract}
In this paper, a fractional-order Chen system is discussed and analyzed by using the frequency-domain approximation method. Its chaotic characteristic can be observed by numerical simulation when the order is as low as 2.9. Then the existence of chaos is verified through the Lyapunov exponent diagrams, bifurcation diagrams, and phase orbits diagrams. At last, based on the fractional-order Chen system, a kind of double encryption algorithm is proposed and adopted to realize the image encryption. The experimental results and analysis show that the proposed method not only has the ideal effect of image encryption, but also has a high security of resisting the statistical attacks. That is, the proposed method is suitable and feasible for image encryption.
\end{abstract}

Keywords: fractional-order; Chen system; Lyapunov exponent diagrams; bifurcation diagrams; image encryption

\section{Introduction}

Although fractional calculus has a history of more than 300 years ${ }^{[1]}$, the applications of fractional calculus to physics and engineer have only become a hot topic in recent years. Since the chaos phenomenon has been found in large number of fractional-order system, the dynamic characteristics of the fractional system has become a research hotspot. Some fractional-order chaotic systems have been proposed and researched, such as the fractional-order Lorenz system ${ }^{[2]}$, the fractional-order Chen system ${ }^{[3-6]}$, the fractional-order of Lü system ${ }^{[7]}$.
With the rapid development of digital and network technology, the significance of information security has arisen more and more attention in recent years. Especially for images, a kind of important information carrier, it may contain much inferable information. Therefore, its security has increasingly been important and become one of research focus in the field of information security. Image encryption technology is an effective method to protect the transmission of digital images ${ }^{[8]}$. So far, many kinds of image encryption technology have been reported, such as text encryption technology ${ }^{[9]}$, quad-tree image encryption technology ${ }^{[10]}$, chaotic encryption technology ${ }^{[11]}$, image encryption 
technology based on DNA ${ }^{[12]}$, and so on ${ }^{[13-15]}$. Intrinsic properties of chaotic systems such as sensitivity to initial conditions, unpredictability, pseudo-randomness, and ergodicity, are considered analogous to an ideal cryptosystem. So the chaotic encryption technology has increasingly highlighted its advantage and become popular in the image encryption. Compared with integer-order chaotic system, Fractional-order chaotic system has larger key space and more complex random sequence ${ }^{[16,17]}$. It is more suitable for image encryption, but the research of image encryption based on fractional-order chaotic system is very few and worthy of further study.

In this paper, a fractional-order Chen system is discussed and analyzed by using approximation of the frequency domain method. Its chaotic characteristic can be observed by numerical simulation when the order is as low as 2.9. Then the existence of chaos is verified through the Lyapunov exponents' diagram, bifurcation diagrams, and phase orbits diagrams. At last, based on this fractional-order Chen system, a kind of double encryption algorithm is proposed and adopted to realize the image encryption. The experimental results and analysis show that the proposed method is effective and practical for image encryption.

\section{Fractional-order Chen system}

The fractional-order Chen system is described as follows ${ }^{[18]}$

$$
\left\{\begin{array}{l}
\frac{d^{\alpha} x}{d t^{\alpha}}=a(y-x) \\
\frac{d^{\beta} y}{d t^{\beta}}=(c-a) x-x z+c y \\
\frac{d^{\gamma} z}{d t^{\gamma}}=x y-b z
\end{array}\right.
$$

Where system parameters $a, b, c \in R, \alpha, \beta$, and $\gamma$ refer to the fractional order. Generally speaking, the positive Lyapunov exponent is an important characteristic to show chaotic behavior of systems. Therefore, the Lyapunov exponents' diagrams and bifurcation diagrams of the fractional-order Chen system are firstly discussed in this paper. When fixing $\alpha=1, \beta=1, \gamma=$ $0.9, b=3, c=28$, and varying $a$, the Lyapunov exponents' diagram and bifurcation diagram of the system are shown in Fig.1 (a), and (b), respectively. From the Fig.1, we can obtain that chaotic behaviors may exist in the system when $a \in[36,47]$. When selecting $\alpha=1, \beta=1, \gamma=0.9, \mathrm{a}=40, \mathrm{c}=28$, and varying $b$, the Lyapunov exponents' diagram and bifurcation diagrams of the system are shown in Fig.2 (a), and (b), respectively. From the Fig.2, we can observe that the system may has chaotic behaviors when $\mathrm{b} \in[0.8,5]$. When fixing $\alpha=1, \beta=1, \gamma=0.9, \mathrm{a}=$ $40, \mathrm{~b}=3$, and varying $\mathrm{c}$, the Lyapunov exponents' diagram and bifurcation diagram of the system are shown in Fig.3 (a), and (b), respectively. From the Fig.3, chaotic behaviors may exist in the system whenc $\in$ $[23,32]$. By the above analysis, we can conclude that chaotic behaviors exist in the fractional-order Chen system when the order is as low as 2.9. Furthermore, when selecting $\alpha=1, \beta=1, \gamma=0.9, a=40, b=3$, and $\mathrm{c}=28$, a chaotic attractor can be obtained by numerical simulation, which is shown in Fig.4. The result is consistent with the theoretical analysis based on the Lyapunov exponents' diagram and bifurcation diagrams. In this paper, we will mainly utilize the fractional-order Chen system with the initial condition of $\alpha=1, \beta=1, \gamma=0.9, a=40, b=3$, and $c=28$. Under the above condition, the system has a typical attractor and can be used in the proposed image encryption algorithm.

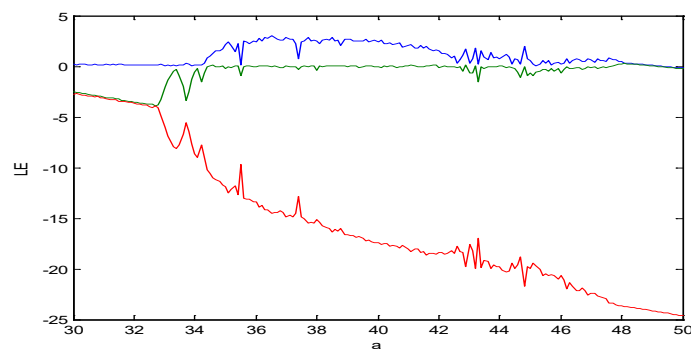

(a) Lyapunov exponents' diagram

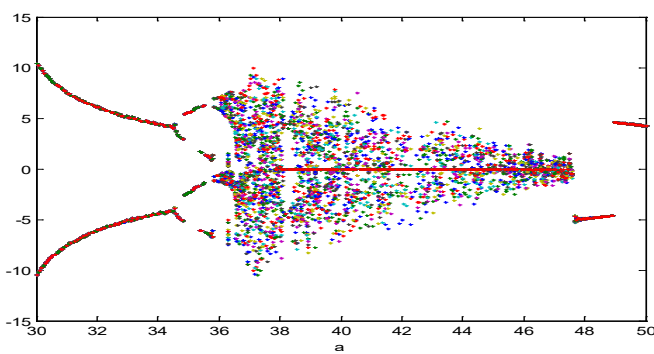

(b) Bifurcation diagram

Fig. 1. Lyapunov exponents' diagram and Bifurcation diagram with $b=3, c=28$, and varying $a$. 


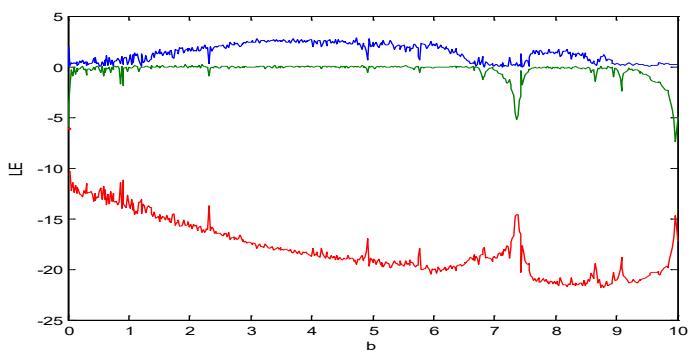

(a) Lyapunov exponents' diagram

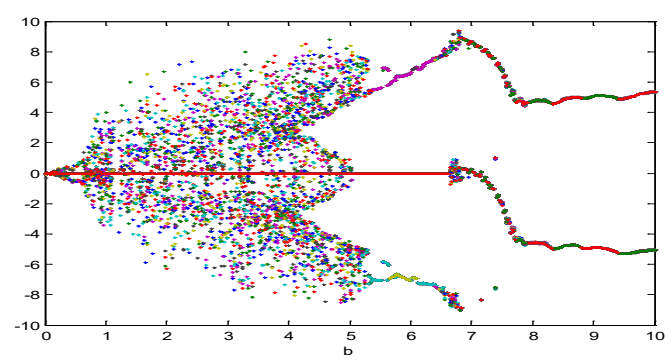

(b) Bifurcation diagram

Fig. 2. Lyapunov exponents' diagram and Bifurcation diagram with $\mathrm{a}=40, \mathrm{c}=28$, and varying $\mathrm{b}$.

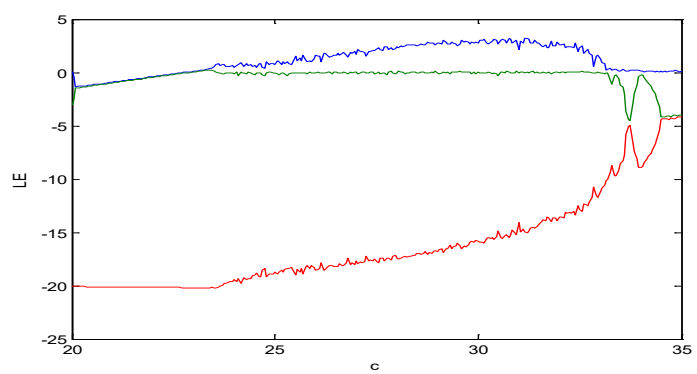

(a) Lyapunov exponentd' diagram

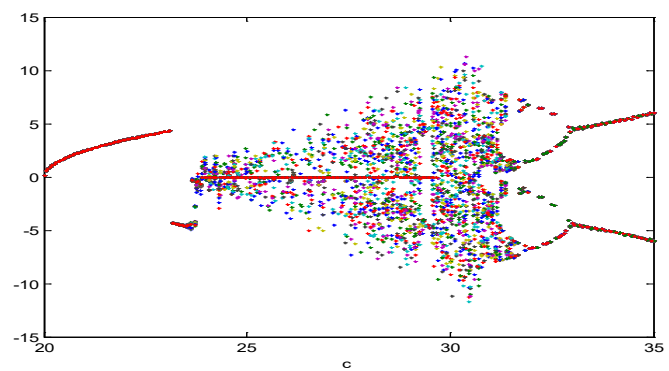

(b) Bifurcation diagram

Fig. 3. Lyapunov exponents' diagram and Bifurcation diagram with $\mathrm{a}=40, \mathrm{~b}=3$, and varying $\mathrm{c}$.

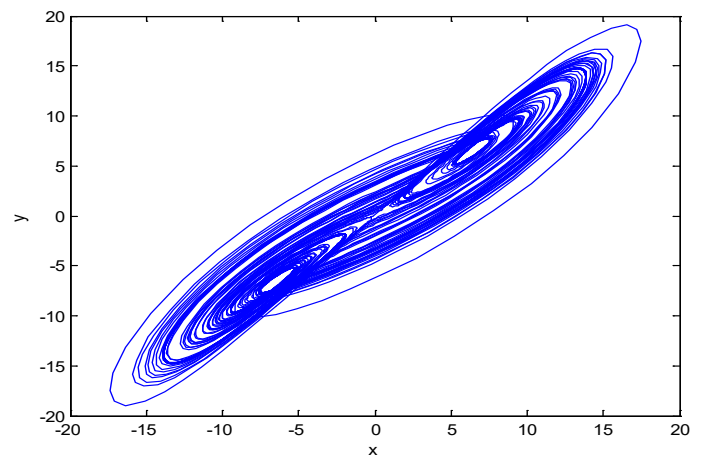

Fig. 4. Chaotic attractor with $a=40, b=3$, and $c=28$.

\section{Image Encryption Algorithm}

The steps of the proposed Algorithm are listed briefly as follows:

Step 1: Generate three chaotic sequences $\mathrm{x}, \mathrm{y}$ and $\mathrm{z}$ by using fraction-order Chen system.

Step 2: Decompose the color image into R, G and B channels.

Step 3: The sequence $\mathrm{x}$ is sorted and used to scramble the matrixes of $R, G$ and $B$ channels using confusion operation to randomly shuffle the pixel position.

Step 4: The three chaotic sequences is combined with scrambled matrixes using diffusion operation to change the pixel value.

Step 5: Obtain the encrypted image.

For decryption process, it is an inverse process of encryption. That is to say, the operation of encryption should be performed reversely.

\section{Experimental Results}

In this paper, a color Image of size $256 * 256$ is selected as the original image shown in Fig.5 (a). The experimental simulation has been carried out when fixing $\alpha=1, \beta=1, \gamma=0.9, a=40, b=3, c=28$, and setting the initial values of state variables $\left(\mathrm{x}_{0}, \mathrm{y}_{0}, \mathrm{z}_{0}\right)$ to $(0.1,0.2,0.3)$. The encrypted image and the decrypted images are shown in Fig.5 (b) and (c), respectively. From the Fig.5, we can observe the encrypted image is completely different from the original image, and become an unrecognizable image. While the decrypted image and the original image is identical. 


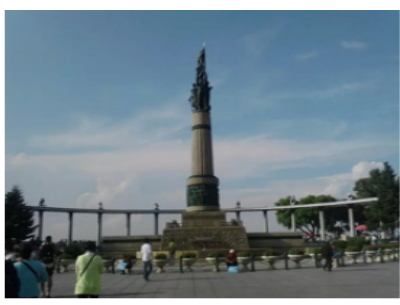

(a)

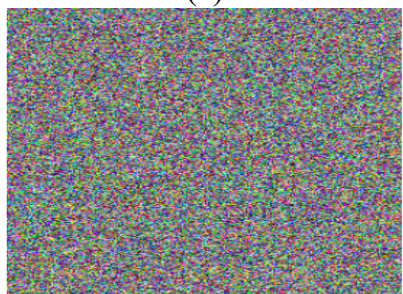

(b)

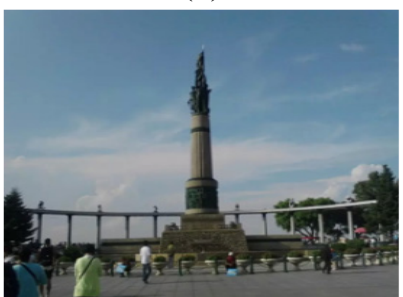

(c)

Fig. 5. The experimental results (a) the original image, (b) the encrypted image (c) the decrypted image.

\section{Performance Analysis}

\subsection{Key sensitivity analysis}

An efficient or an ideal encryption scheme should have a high security; That is, it should be very sensitive to the secret key. It means a tiny change in the key will lead to a significant change in the encrypted image, even the decryption will fail when using the secret key with a slight change. In order to validate the key sensitivity, a test is performed using a key that is a bit different from the original key to decrypt the original image. The decrypted results are shown in Fig.6 (a), (b), (c), and (d), respectively. Fig.6 (a), (b), and (c) show the decrypted images with the deviation of any one of the state variables values $\mathrm{x}_{0}, \mathrm{y}_{0}$ and $\mathrm{z}_{0}$ is up to the $10^{-9}$, and keep others unchanged. Fig. 6 (d) shows the decrypted image with the system parameter $\mathrm{a}=40.000000001$, and keep others unchanged. It is evident that the decryption is failed as long as there is a tiny change of the original key. So it can be concluded that the proposed algorithm has high sensitivity to the secret key.

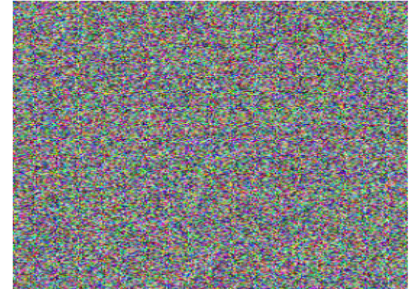

(a)

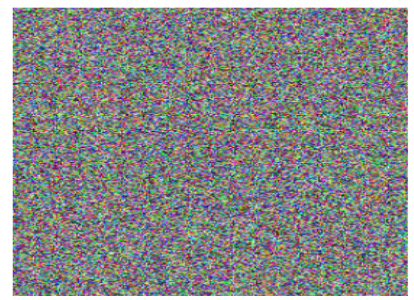

(b)

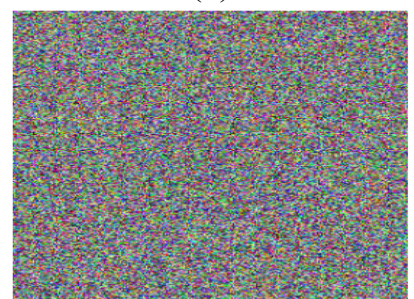

(c)

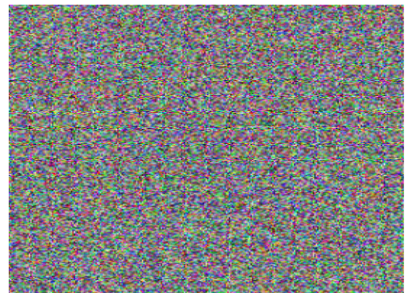

(d)

Fig. 6. Decrypted image with (a) incorrect $\mathrm{x}_{0}$, (b) incorrect $\mathrm{y}_{0}$, (c) incorrect $z_{0}$, (d) incorrect system parameter $a$.

\subsection{Histogram analysis}

Generally speaking, histogram can clearly reflect the distribution of pixel values in the image, so it is convenient for people to correctly evaluate the pixel values distribution of an image. Here the histograms of the original image are illustrated in Fig.7 (a), (b), and (c), respectively. The histograms of the encrypted image are illustrated in Fig.8 (a), (b), and (c), respectively. It can be seen that the histograms of the encrypted image are flat enough, and uniformly distributed. It indicates that no useful information can be extracted about the original image. Thus, we can conclude that the proposed 
encryption method have good resistance against statistical attack.

\subsection{Correlation Coefficient analysis}

Correlation coefficient is an important indicator to measure the relevancy between two adjacent pixels. The correlation coefficient is generally high in the original image. That means it is vulnerable to statistical attack. An image encryption should decrease the correlation coefficient in the encrypted image as much as possible. Here, we calculate correlation coefficient for an image according to the following formula [19-21]:

r_xy $=(\operatorname{cov}(\mathrm{x}, \mathrm{y})) /(\sqrt{ }(\mathrm{D}(\mathrm{x})) \sqrt{ }(\mathrm{D}(\mathrm{y})))$,

where

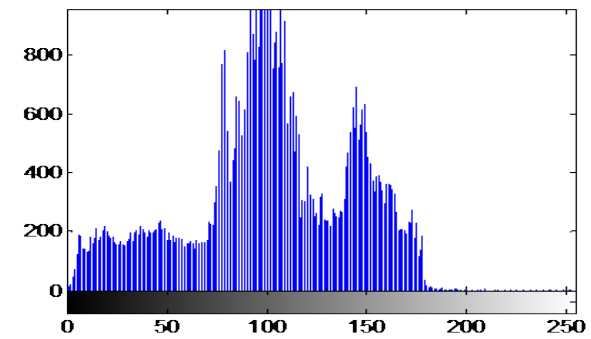

(a)

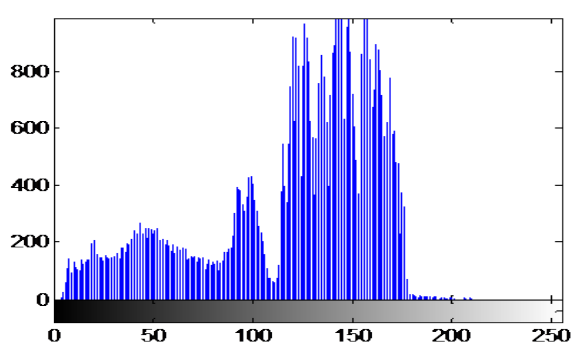

(b)

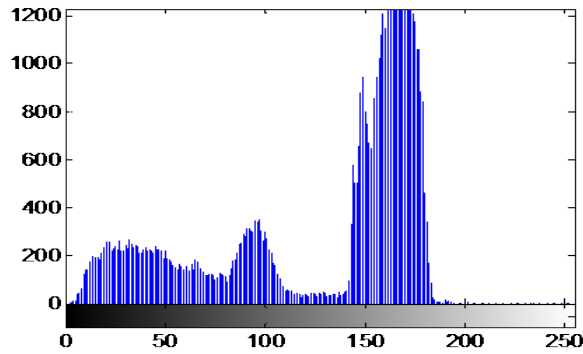

(c)

Fig. 7. Histograms of the original image (a) R channel, (b) G channel, and (c) B channel. $\operatorname{cov}(\mathrm{x}, \mathrm{y})=\frac{1}{\mathrm{~N}} \sum_{\mathrm{i}=1}^{\mathrm{N}}\left(\mathrm{x}_{\mathrm{i}}-\mathrm{E}(\mathrm{x})\right)\left(\mathrm{y}_{\mathrm{i}}-\mathrm{E}(\mathrm{y})\right)$,

$\mathrm{E}(\mathrm{x})=\frac{1}{\mathrm{~N}} \sum_{\mathrm{i}=1}^{\mathrm{N}} \mathrm{x}_{\mathrm{i}}$,

$\mathrm{D}(\mathrm{x})=\frac{1}{\mathrm{~N}} \sum_{\mathrm{i}=1}^{\mathrm{N}}\left(\mathrm{x}_{\mathrm{i}}-\mathrm{E}(\mathrm{x})\right)^{2}$,

The correlation coefficients of the original image and the encrypted image are provided in Table 1. It can be seen from Tabel 1 that the correlation coefficient of the original image is close to one, while that of the encrypted image is close to zero. It is obvious that the adjacent pixels in encrypted image are almost uncorrelated. So, we can obtain that the proposed encryption method has good resistance against statistical attack.

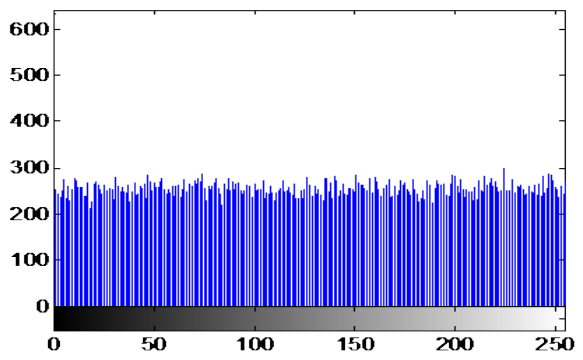

(a)

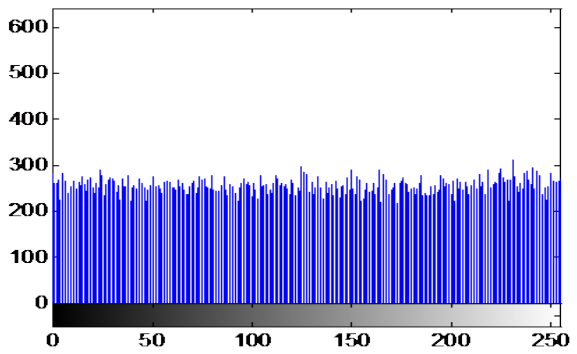

(b)

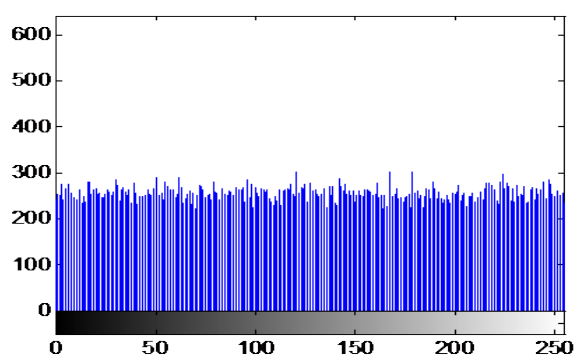

(c)

Fig. 8. Histograms of the encrypted image (a) R channel, (b) G channel, and (c) B channel. 
Table 1 Correlation Coefficient

\begin{tabular}{|l|l|l|}
\hline & Original Image & Encrypted Image \\
\hline$R$ & 0.95176 & 0.0025015 \\
\hline$G$ & 0.9591 & 0.0014247 \\
\hline$B$ & 0.97131 & 0.0012004 \\
\hline
\end{tabular}

\section{Conclusion}

This paper firstly discusses and analyzes a fractionalorder Chen system. Its chaotic characteristic can be observed by numerical simulation when the order is as low as 2.9. Then the existence of chaos is verified through the Lyapunov exponents' diagram, bifurcation diagrams, and phase orbits diagrams. At last, based on the fractional-order Chen system, a kind of double encryption algorithm is proposed and adopted to realize the image encryption. The experimental results and analysis show that the proposed method has good encryption effect, and high sensitivity to the secret key. It also has good resistance against statistical attack. Besides, the encryption process is simple, and it is convenient to encrypt image. So the proposed algorithm is feasible and effective, and it is suitable for the image encryption field.

\section{Acknowledgements}

This work was supported in part by the Young Scientists Fund of the National Natural Science Foundation of China (Grant No.11202148, 61174094).

\section{References}

1. G Ilia, and G Elena, Chaotic dynamics of the fractional Lorenz system, Phys. Rev. Lett. 91(3) (2003) 034101034101.

2. Yu Yongguang, Li Hanxiong, Wang Sha, and Yu Junzhi, Dynamic analysis of a fractional-order Lorenz chaotic system, Chaos, Solitons and Fractals. 42(2) (2009) 11811189.

3. Lu Junguo, Nonlinear observer design to synchronize fractional-order chaotic systems via a scalar transmitted signal, Physica A. 35 (2006) 107-118.

4. Lu Junguo, and Chen Guanrong, A note on the fractionalorder Chen system, Chaos, Solitons and Fractals. 27(3) (2006) 685-688

5. Li Changpin, and Peng Guojun, Chaos in Chen's system with a fractional order, Chaos, Solitons and Fractals. 22(2) (2004) 443-450.
6. Li Chunguang, and Chen Guanrong, Chaos in the fractional order Chen system and its control, Chaos, Solitons and Fractals. 22(3) (2004) 549-554.

7. Lu Junguo, Chaotic dynamics of the fractional-order $\mathrm{Li}$ system and its synchronization, Physics Letters A. 354(4) (2006) 305-311.

8. Zhang Xiaoyan, Wang Chao, and Sun Zhiren, et al. An image encryption scheme based on sequential CA, Optics and Precision Engineering. 16(9) (2008) 1781-1786.

9. Nooshin B, Youset F, and Karim A, A robust hybrid method for image encryption based on Hopfield neural network, Computer \& Electrical Engineering. 38(2) (2012) 356-369.

10. Liu Lili, Zhang Qiang, and Wei Xiaopeng, A RGB image encryption algorithm based on DNA encoding and chaos map, Computer and Electrical Engineering. 38(5) (2012) 1240-1248.

11. Zhang Yonghong, and Zhang Bo, Algorithm of image encrypting based on logistic chaotic system, application research of Computer. 32(6) (2015) 1770-1773

12. Zhang Jian, and Fang Dongxin, Image encryption technology applied chaotic maps index and DNA coding, Computer Engineering and Design. 36(3) (2015) 613-618

13. Wang Zhen, Huang Xia, Li Ning, and Song Xiaona, Image encryption based on a delayed fractional-order chaotic logistic system, Chin. Phys. B. 21(5) (2012) 050506-050511

14. Peng Zaiping, Wang Chunhua, Lin Yuan, and Luo Xiaowen, A novel four-dimensional multi-wing hyperchaotic attractor and its application in image encryption, Acta Phys. sin. 63(24) (2014) 240506-240515.

15. Liao Qinan, Lu Shoudong, and Sun Xianbo, Digital image encryption algorithm by combining hyper chaotic sequences and shift cipher, Journal of Chinese Computer Systems. 36(2) (2015) 332-337.

16. Kanso, A., and Ghebleh, M., A novel image encryption algorithm based on a 3D chaotic map, Commun. Nonlinear Sci.Numer. Simul. 17(7) (2012) 2943-2959.

17. Ye,,G.D., and Wong, K.W., An image encryption scheme based on time-delay and hyperchaotic system, Nonlinear Dyn. 71(1-2) (2013) 259-267.

18. Li Chunguang, and Chen Guanrong, Chaos in the fractional order Chen system and its control, Chaos, Solitons and Fractals. 22(3) (2004) 549-554.

19. Huang Xiaoling, Image encryption algorithm using chaotic chebyshev generator, Nonlinear Dyn . 67(4) (2012) 2411-2417.

20. S. Behnia, A. Akhshani, S. Ahadpour, H. Mahmodi, and A. Akhavan,, A fast chaotic encryption scheme based on piecewise nonlinear chaotic maps, Phys. Lett. A. 366(4-5) (2007) 391-396.

21. R. Rhouma, S.Meherzi, and S.Belghith, OCML-based color image encryption, Chaos Solitons Fractals. 40(1) (2009) 309-318. 